\title{
Real-time gait metric estimation for everyday gait training with wearable devices in people poststroke
}

\author{
Philipp Arens ${ }^{1,2}$, Christopher Siviy ${ }^{1,2}$ (D) Jaehyun Bae ${ }^{1,2}$, Dabin K. Choe ${ }^{1,2}$, Nikos Karavas ${ }^{1,2}$, \\ Teresa Baker ${ }^{3}$, Terry D. Ellis ${ }^{3}$, Louis N. Awad ${ }^{1,2,3}$ and Conor J. Walsh ${ }^{1,2, *}$ \\ 'John A. Paulson School of Engineering and Applied Sciences, Harvard University, Cambridge, Massachusetts, USA \\ ${ }^{2}$ Wyss Institute for Biologically Inspired Engineering, Harvard University, Cambridge, Massachusetts, USA \\ ${ }^{3}$ Department of Physical Therapy and Athletic Training, Boston University, Boston, Massachusetts, USA \\ *Corresponding author: Email.walsh@seas.harvard.edu \\ Philipp Arens and Christopher Siviy contributed equally to this work.
}

Received: 1 May 2020; Revised: 1 September 2020; Accepted: 29 September 2020

Key words: performance characterisation; exoskeletons; exosuits

\begin{abstract}
Hemiparetic walking after stroke is typically slow, asymmetric, and inefficient, significantly impacting activities of daily living. Extensive research shows that functional, intensive, and task-specific gait training is instrumental for effective gait rehabilitation, characteristics that our group aims to encourage with soft robotic exosuits. However, standard clinical assessments may lack the precision and frequency to detect subtle changes in intervention efficacy during both conventional and exosuit-assisted gait training, potentially impeding targeted therapy regimes. In this paper, we use exosuit-integrated inertial sensors to reconstruct three clinically meaningful gait metrics related to circumduction, foot clearance, and stride length. Our method corrects sensor drift using instantaneous information from both sides of the body. This approach makes our method robust to irregular walking conditions poststroke as well as usable in real-time applications, such as real-time movement monitoring, exosuit assistance control, and biofeedback. We validate our algorithm in eight people poststroke in comparison to lab-based optical motion capture. Mean errors were below $0.2 \mathrm{~cm}(9.9 \%)$ for circumduction, $-0.6 \mathrm{~cm}(-3.5 \%)$ for foot clearance, and $3.8 \mathrm{~cm}(3.6 \%)$ for stride length. A single-participant case study shows our technique's promise in daily-living environments by detecting exosuit-induced changes in gait while walking in a busy outdoor plaza.
\end{abstract}

\section{Introduction}

Stroke is a prominent cause of long-term disability affecting over seven million people in the United States alone (Benjamin et al., 2019), many of whom suffer from hemiparesis. Though the impact of hemiparesis is wide-ranging, its effects are particularly apparent in gait; hemiparetic gait is typically slow, asymmetric, and inefficient (Olney and Richards, 1996), posing an increased risk of fall. These impairments can limit community ambulation in a way that significantly affects participation in activities of daily living (ADLs), often leading to isolation, depression, and even secondary strokes (Do Lee et al., 2003; Linden et al., 2007).

Rehabilitation after stroke plays a crucial role in restoring gait. Comprising an iterative process alternating between gait assessment and intervention such as motor recovery training, extensive literature demonstrates that effective gait training after stroke is functional, task-specific, intensive, and repetitive (Eng and Tang, 2007; Langhorne et al., 2009; Winstein et al., 2016). Furthermore, deterioration in regained 
mobility after inpatient care is a major challenge for effective rehabilitation, emphasizing the importance of continued, postdischarge training throughout the chronic stages of stroke recovery (Paolucci, 2008).

\section{Gait Monitoring and Wearable Devices in Poststroke Rehabilitation}

To encourage such training paradigms in and beyond the clinic, our group develops wearable robots called soft exosuits (see Figure 1) that deliver mechanical power to the paretic (P) ankle via functional apparel and cable-based actuation systems, in step with a patient's natural gait cycle (Awad et al., 2017; Bae et al., 2018). During activities typical to rehabilitation, soft exosuits have been found to reduce common gait compensations such as hip hiking, circumduction, and propulsion asymmetry (Awad et al., 2017)locomotor improvements that may amplify benefits from conventional training (Awad et al., 2020). Moreover, a unique characteristic of soft exosuits is their ability to switch quickly from applying assistance to no assistance (while minimally restricting gait), potentially enabling new approaches for gait rehabilitation. Beyond soft exosuits, other lightweight wearable robots have improved ankle power production (McCain et al., 2019) and walking speed (Jayaraman et al., 2019) in people poststroke.

While these outcomes are promising, monitoring gait improvements after stroke can be challenging, particularly in outpatient or community-based rehabilitation environments. Clinical tools, like mobility scales and outcome measures that support therapists in assessing ADL-related improvements, are reliable in classifying functional abilities. Still, they may lack the precision and frequency required to reflect subtle changes in mobility (Toro et al., 2007), such as those induced by an exosuit. Similarly, even highly trained clinicians may have difficulty visually detecting stride-by-stride or between-session improvements (Paolucci et al., 2001; Walker et al.,2013). Moreover, in community or at-home rehabilitation, insurance status, restricted patient mobility, or remote living conditions may constrain access to regular physical therapy assessment altogether (Hickey et al., 2012).
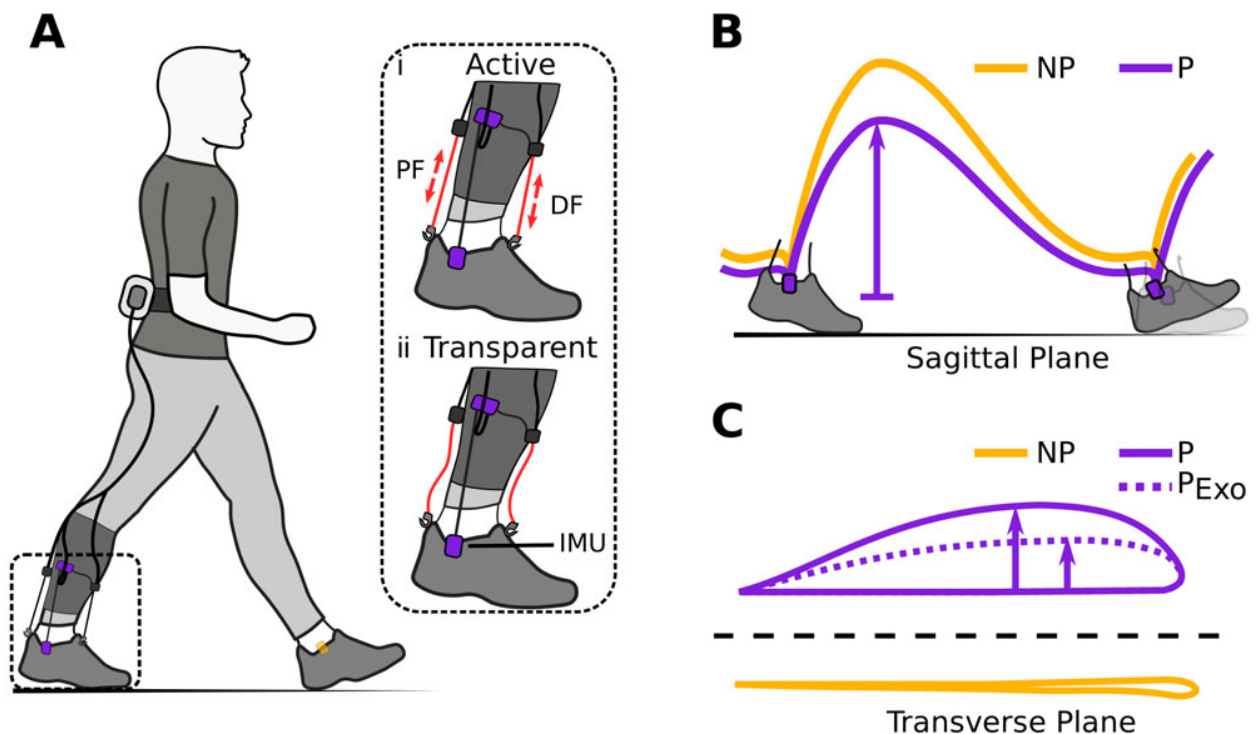

Figure 1. (a) Hardware setup for ambulatory gait monitoring in exosuit-assisted walking. The integrated foot inertial measurement units analyze the gait cycle to inform the exosuit controller when to correctly provide plantarflexion (PF) and dorsiflexion (DF) assistance (i). The exosuit can quickly switch from active to transparent mode (ii) allowing a person to walk without assistance and hence their natural walking pattern if desired. Typical impairments in poststroke gait include reduced ground clearance (b), often causing individuals to compensate with increased lateral displacement during swing (c). Awad et al. (2017) showed that exosuit assistance can help reduce such compensatory mechanisms. 
Instrumented ambulatory monitoring may help overcome some of these barriers by allowing clinicians to assess gait remotely, objectively, and continually. In particular, integration with new rehabilitation approaches such as telemedicine could help extend clinical gait training to the community on a broader scale. Furthermore, in combination with new wearable robot technologies such as soft exosuits, quantitatively monitoring the immediate impact of provided assistance may allow clinicians to adjust training routines and tune assistance parameters effectively within an in-person or remote training session. Similarly, recent studies suggest that wearable robots can tune assistance based on physiological data (Zhang et al., 2011). Likewise, future iterations of soft exosuits could automatically tune assistance based on an individual's gait metric response by integrating ambulatory gait metrics directly into the exosuit's control loop.

Indeed, being small, lightweight, and unobtrusive, inertial measurement units (IMUs) in particular have proven attractive for estimating spatiotemporal gait metrics in various clinical and nonclinical applications. Soft exosuits, for instance, use IMUs as a high-level control input for temporal gait segmentation. Necessarily, this has enabled exosuits to export a variety of clinically important temporal gait metrics such as stance-swing ratios and P and nonparetic (NP) step time (Awad et al., 2017). Outside the field of wearable robotics, a number of studies investigating IMUs for gait metric reconstruction over the last decade have shown promising outcomes both in healthy (Sabatini et al., 2005; Schepers et al., 2010; Mariani et al., 2010; Köse et al., 2012; Rebula et al., 2013; Benoussaad et al., 2016) and impaired (Trojaniello et al., 2014; Rampp et al., 2015; Ferrari et al., 2016; Visi et al., 2017; Porciuncula et al., 2018) populations. Though work relying solely on IMUs in people poststroke has been more limited, some studies have also explored approaches complementing IMUs with additional sensors such as foot switches and ultrasound sensors (Meulen et al., 2016) that can directly provide inter-limb measures such as step length. Yet other studies incorporate kinematic models combined with IMUs spread across the lower limbs to compute gait metrics (Moore et al., 2017; Wang et al., 2018). Please see the section "Correcting Sensor Drift" for a more detailed explanation of the methods used in the literature. Broadly, though encouraging, existing methods often rely on manual tuning that may not be adaptable across highly heterogeneous populations such as people poststroke. A requirement of manual tuning may limit these methods' usability for control in wearable robots such as exosuits. Motivated by these factors, our goal was to update exosuit gait detection algorithms developed for poststroke populations without adding additional hardware requirements. Moreover, it is to date unclear if IMU-based gait monitoring is accurate even in the presence of potential motion artifacts from exosuit assistance.

In this paper, we present an accurate and robust method of computing gait metrics in poststroke walking with IMUs attached bilaterally to the paretic and nonparetic foot. As a secondary objective, we show a proof of concept for using gait monitoring integrated with an exosuit in the community. Though we anticipate this method is effective with or without an assistive device, to avoid imposing additional burdens on clinicians wishing to monitor gait in their patients wearing exosuits, we use IMUs already integrated into an exosuit system.

\section{Algorithm for Robust Zero-Velocity Update Detection}

Estimating spatial gait metrics relies on reconstructing foot trajectories. As IMUs measure linear accelerations (and angular velocities), measuring changes in position typically requires numerical integration of sensor signals. However, due to sensor inaccuracies, estimation error (i.e., drift) accumulates during numerical integration, rendering the estimated metrics unusable within seconds if not accounted for. Canonically, these inaccuracies manifest themselves in the form of bias ( $\beta$; poor estimates in the signal mean) and noise ( $\epsilon$; zero-mean inaccuracies) (Woodman, 2007).

\section{Correcting Sensor Drift}

State-of-the art ambulatory gait monitoring systems correct this drift by discarding estimation error using zero-velocity updates (ZUPTs); recurring instances or periods in the gait cycle during which foot motion is 
known to be zero (Foxlin, 2005). Conventionally, approaches look at either linear accelerations, angular velocities, or combinations of both signals to detect ZUPTs (Skog et al., 2010). That is, researchers use empirically tuned thresholds to determine when the designated ZUPT detection signals fall reasonably close to zero. Additional constraints such as mandatory ZUPT window lengths are also common to improve detection accuracy and increase robustness (Jim, 2009; Rampp et al., 2015; Rebula et al., 2013). Typically, when the ipsilateral detection signals approach zero-suggesting the (ipsilateral) foot is stationary-algorithms restart the integration. This constrains the time period in which error can accumulate and, by modeling the error propagation over time, discards already accumulated drift from the faulty position estimate. Such approaches have successfully estimated gait parameters in unimpaired individuals (Sabatini et al., 2005) as well as in various populations with gait pathologies (Mariani et al., 2013; Trojaniello et al., 2014; Ferrari et al., 2016).

While promising, variability in stroke induced hemiparetic gait, especially on the paretic side (Kempski et al., 2018), can make it difficult to generalize thresholds for inter- and even intra-patient use; unexpected or irregular foot motion may lead to missed or unwarranted ZUPT detections if thresholds are not optimally tuned, a complication that may result in highly inaccurate gait metric estimates (Rebula et al., 2013). Other studies (Schepers et al., 2007; Schepers et al. 2010) rely on additional sensors such as foot switches to segment gait and define ZUPTs. Furthermore, there is no work to our knowledge demonstrating that IMU-based gait monitoring can resolve small improvements in walking on an intraperson basis in community environments.

\section{Zero-Velocity Update Detection Using Contralateral Foot Information}

We implemented our ZUPT detection method using a strap-down double integration algorithm with linear drift compensation similar to Sabatini et al. (2005). The gait segmentation uses information from both feet to identify paretic toe-off and nonparetic toe-off. As the toe-off event on the contralateral side indicates early single limb support on the ipsilateral side, biomechanically this event is likely to coincide with a foot-flat instance of the ipsilateral foot, assuming the person is not running. Commonly, paretic-foot instability can introduce local minima around the sharp negative peaks in Figure 2 that correspond with toe-off. For realtime applications, waiting until angular velocity switches from negative to positive helps avoid these local minima. As this zero crossing follows the actual (peak) toe-off event in the gait cycle by approximately 4 $6 \%$, it should be noted that the ZUPT instance will show the same degree of latency compared to the actual (biomechanical) toe-off event, resulting in a slight shift from early stance toward mid-stance (see Figure 2). Confirming contralateral toe-off with the zero crossing of contralateral angular velocity, we leverage this biomechanical constraint to reliably define ZUPT instances on the opposite, ipsilateral foot.

\section{Double Integration Framework}

Figure 3 illustrates the general algorithm framework. All input signals utilized by the algorithmcomprising three-axis accelerations in the local (or sensor) frame, three-axis orientation expressed as unit quaternions computed directly by the onboard IMU Kalman Filter as well as angular velocity in the sagittal plane - are obtained from the exosuit-integrated foot IMUs.

For each time sample, the local acceleration signal is transformed into world frame coordinates based on the current IMU-provided orientation estimate. After removing gravity, the resulting acceleration signal is integrated twice using trapezoidal integration to obtain position estimates. Once the algorithm receives a ZUPT confirmation flag, the integration is re-initialized, and after subtracting the accumulated sensor drift from the uncorrected position estimate we obtain drift compensated position trajectories in each world frame direction. That is, assuming (quasi) constant bias $\beta$ and zero-mean noise $\epsilon$, we can model the measured acceleration as $\hat{a}(t)=a(t)+\beta+\epsilon(t)$, where $a(t)$ refers to the actual acceleration. Consequently, integrating the bias term twice results in position error growing quadratically over time. Knowing the true velocity at each ZUPT event to be zero, the position error $e_{p}$ accumulated over the stride interval $T_{s}$ can be modelled as: 


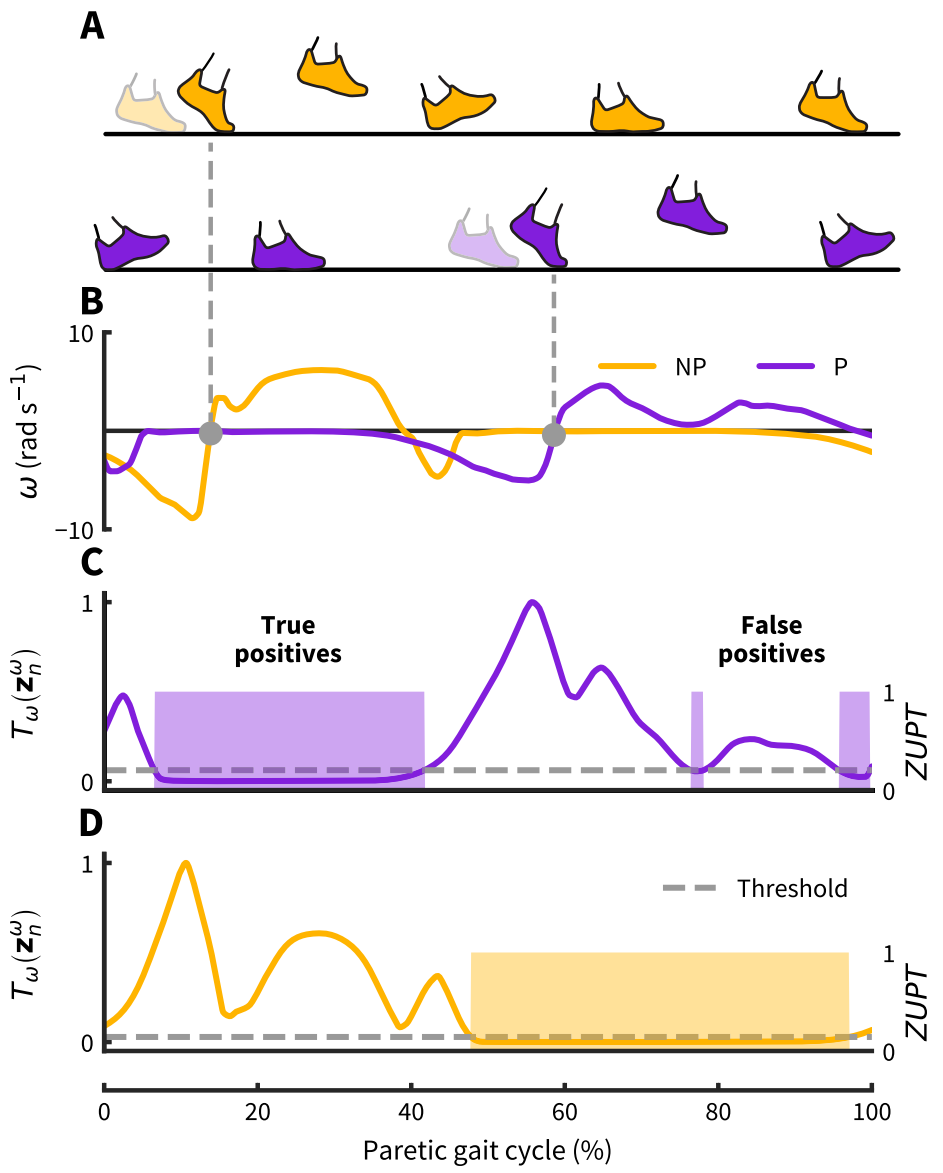

Figure 2. Zero-velocity update detection approach: (a) Nonparetic and paretic gait cycle. (b) Our approach uses contralateral angular velocity zero crossings to detect ZUPT instances on the ipsilateral foot (grey dots). (c-d) Threshold based ZUPT detection shown for an angular rate energy detector as in Skog et al. (2010). This method defines a ZUPT as any time the decision metric $T_{\omega}$ (normalized as shown here) falls below a predetermined threshold. For this particular detector, the decision metric $T_{\omega}$ broadly measures foot rotational energy normalized to vary between 0 and 1. For the demonstrated threshold, the paretic side shows two falsely identified ZUPT phases caused by unanticipated low angular velocity during swing. The same threshold works correctly for the nonparetic side. Both thresholds are normalized to the respective maximum detector signal value.

$$
e_{p}=\iint_{0}^{T_{s}} \beta \mathrm{d} t \mathrm{~d} t=\frac{1}{2} v_{\mathrm{ZUPT}} T_{s},
$$

where $v_{\text {ZUPT }}$ refers to the velocity error (i.e., the estimated velocity) at each ZUPT event.

\section{Gait Metric Computation}

Based on the drift-corrected position trajectories, we computed gait metrics between subsequent ZUPTs. Stride Length (SL) was defined as the transverse-plane distance between two successive ZUPT events of the same foot (i.e., the magnitude of a vector pointing in the direction of progression): 


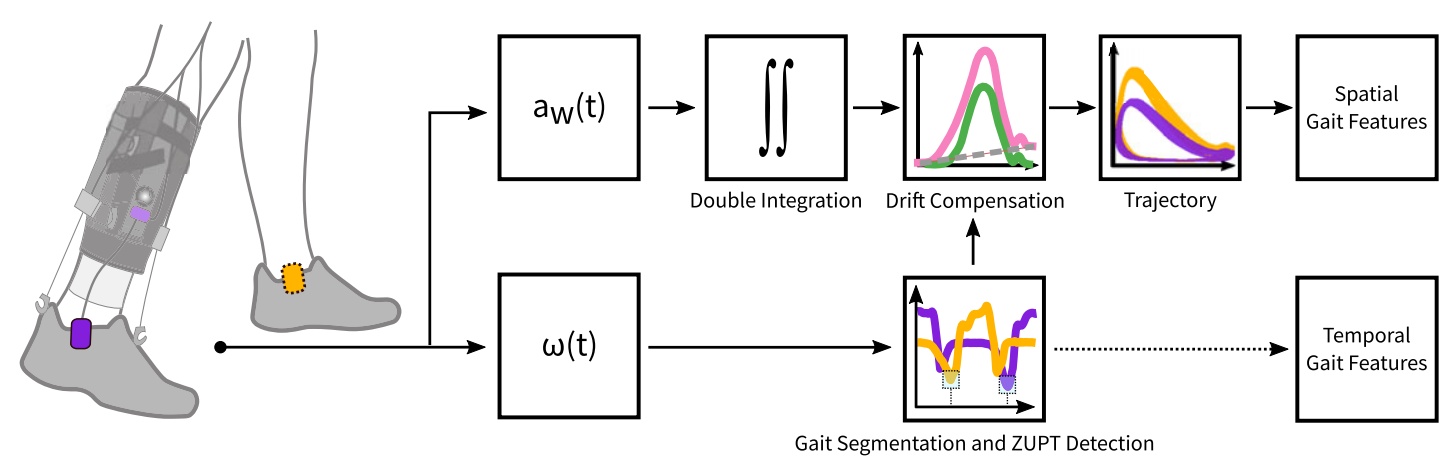

Figure 3. General algorithm pipeline. The exosuit-integrated foot inertial measurement units provide three-dimensional linear, sensor frame acceleration as well as angular velocity. After transforming the acceleration signal to a fixed world frame and removing gravity, the resulting acceleration signal $a_{w}(t)$ is integrated twice to obtain position estimates. The angular velocity is used by the gait detection algorithm to segment the gait cycle - providing temporal gait features - and detect ZUPT instances that allow us to compensate drift from the computed position trajectories. Based on the resulting three-dimensional foot trajectories, spatial gait metrics for both feet can be computed.

$$
S L(i)=\sqrt{d_{x}(i)^{2}+d_{y}(i)^{2}}
$$

where $d_{x}(i)$ and $d_{y}(i)$ refer to the overall displacements within the current (ith) stride in $x$ and $y$ directions, respectively. Maximum lateral displacement (MLD) was computed as:

$$
M L D(i)=\max _{k} D_{x y, i}(k)
$$

where $D_{x y, i}(k)$ marks the perpendicular distance at every sample point $k$ between the direction of progression and the transverse-plane foot trajectory. Similarly, maximum vertical displacement (MVD) was defined as the maximum value of the vertical (z-direction) trajectory. Ground truth motion capture measurements were computed in the same way, using markers rigidly attached to each IMU.

\section{Experimental Validation with a Soft Exosuit}

To determine algorithm performance in terms of accuracy and robustness, we conducted an overground, in-lab study with eight participants in the chronic phase of poststroke recovery. Figure 4 shows the experimental setup. The soft exosuit's design is detailed in Bae et al. (2018), but briefly it consists of a proximally mounted actuation unit connected to a distally mounted textile component at the shank with a pair of Bowden cables, one each for plantarflexion (PF) and dorsiflexion (DF). The Bowden cables' outer sheaths anchor on the shank while their inner cables extend to the foot, generating either PF or DF moments when the respective cable retracts. An IMU (MTi-3; XSens; Netherlands; $100 \mathrm{~Hz}$ ) mounted on either shoe detects the events described in section "Double Integration Framework."

\section{Experimental Overview}

Participants $(N=8$; age: $48.9 \pm 10.0$ years; 4 male; Table 1$)$ wearing the exosuit as described previously, were asked to perform two 4 min bouts on an overground track instrumented with nine force plates (FP4060-15; Bertec; Columbus, OH; 2,000 Hz) and a total length of $36.9 \mathrm{~m}$, one bout with the suit active and one bout with the suit inactive at a comfortable walking speed. The order of the two conditions was randomized and exosuit assistance was set as in Bae et al. (2018), with a peak PF cable force of $25 \%$ body 
A

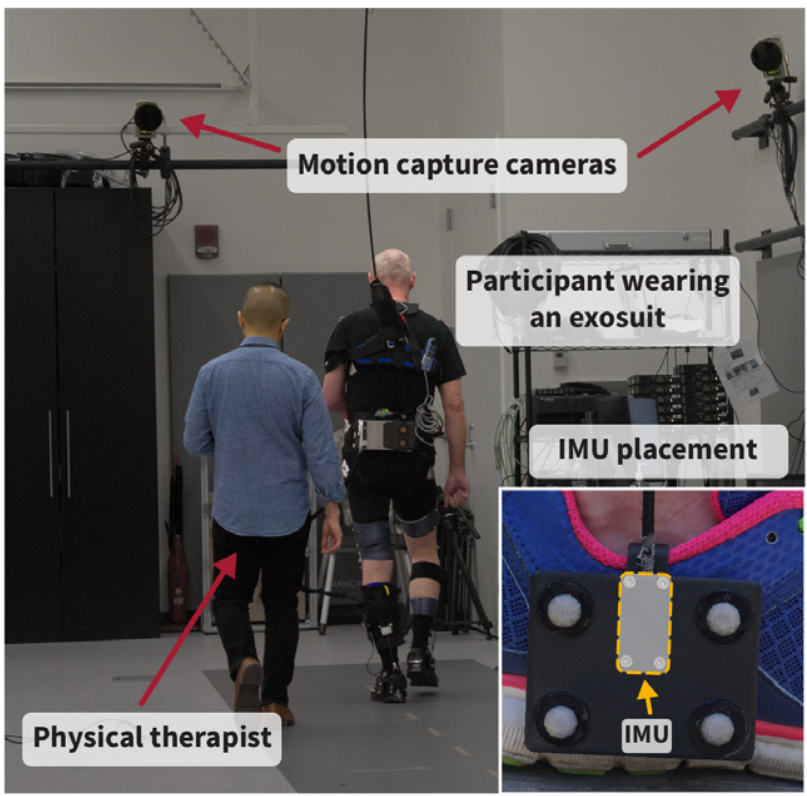

B

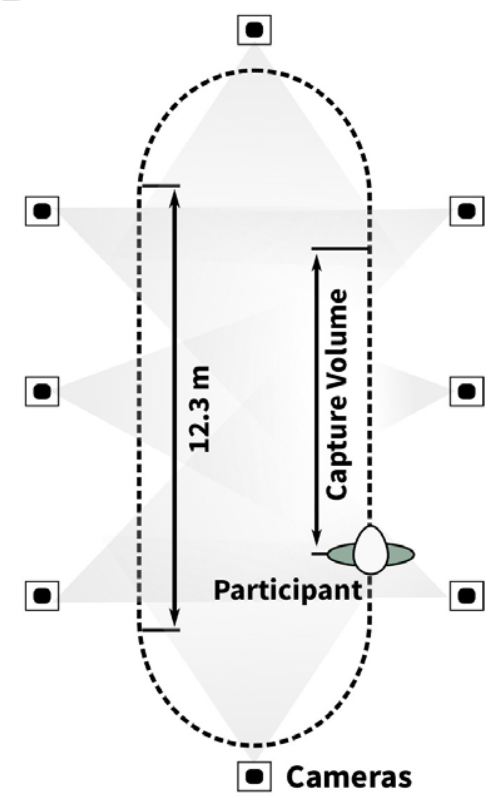

Figure 4. (a) Experimental setup for in-lab validation. Participants wearing an exosuit walked continuously with and without exosuit assistance for 4 min each on an overground track in the motion capture laboratory at Harvard University with a total length of $36.9 \mathrm{~m}$. The inertial measurement units (IMUs) were rigidly attached to marker clusters mounted laterally to both feet to minimize relative movement between the IMU and optical markers (see close-up image). The IMU coordinate frame origin was approximated as the center of the four optical markers. Optical motion capture data were collected during the straight path section that fully lies within the capture volume of the cameras (b).

Table 1. Demographic and clinical information of the participants for the present study, including the paretic side, gender, age, time since stroke (TSS), type of stroke, 10-m walk test speed at a self-selected comfortable pace (10MWS CWS), and the number of usable strides from each participant on the $P$ and NP sides

\begin{tabular}{|c|c|c|c|c|c|c|c|c|}
\hline \multirow[b]{2}{*}{ Participant } & \multirow[b]{2}{*}{ Paretic side } & \multirow[b]{2}{*}{ Gender } & \multirow[b]{2}{*}{ Age (years) } & \multirow[b]{2}{*}{ TSS (years) } & \multirow[b]{2}{*}{ Type of stroke } & \multirow[b]{2}{*}{ 10MWS CWS $(\mathrm{m} / \mathrm{s})$} & \multicolumn{2}{|c|}{ Strides } \\
\hline & & & & & & & $\mathrm{P}$ & NP \\
\hline S1 & $\mathrm{R}$ & M & 35 & 5.6 & Hemorrhagic & 1.45 & 38 & 39 \\
\hline $\mathrm{S} 2$ & $\mathrm{R}$ & M & 56 & 4.2 & Hemorrhagic & 0.68 & 48 & 46 \\
\hline S3 & $\mathrm{R}$ & $\mathrm{F}$ & 34 & 11.1 & Hemorrhagic & 1.15 & 24 & 22 \\
\hline S4 & $\mathrm{L}$ & $\mathrm{F}$ & 58 & 19.0 & Ischemic & 0.79 & 10 & 9 \\
\hline S5 & $\mathrm{R}$ & $\mathrm{F}$ & 50 & 8.3 & Hemorrhagic & 1.72 & 12 & 13 \\
\hline S6 & $\mathrm{L}$ & M & 61 & 8.5 & Hemorrhagic & 0.78 & 42 & 45 \\
\hline S7 & $\mathrm{L}$ & $\mathrm{F}$ & 50 & 5.2 & Ischemic & 0.96 & 27 & 22 \\
\hline S8 & $\mathrm{L}$ & M & 47 & 14.2 & Ischemic & 0.74 & 26 & 24 \\
\hline
\end{tabular}

Abbreviations: NP, nonparetic; P, paretic.

weight and peak DF cable position held constant after tuning by an experienced clinician. Accuracy was determined with respect to a 14-camera optical motion capture system (Oqus; Qualisys; Sweden; $200 \mathrm{~Hz}$ ). For synchronization purposes, data were streamed via Bluetooth connection to a nearby computer and analyzed through postprocessing. Medical clearance and signed informed consent forms approved by the Harvard University Human Subjects Review Board were obtained for all participants. 


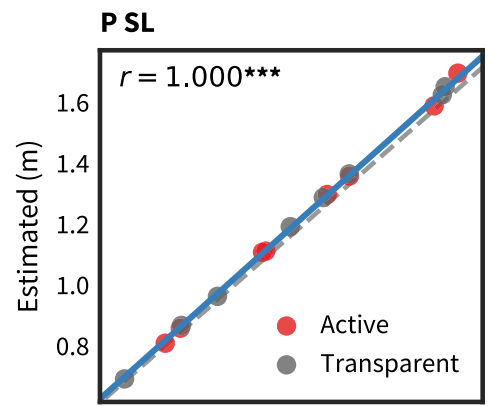

NP SL

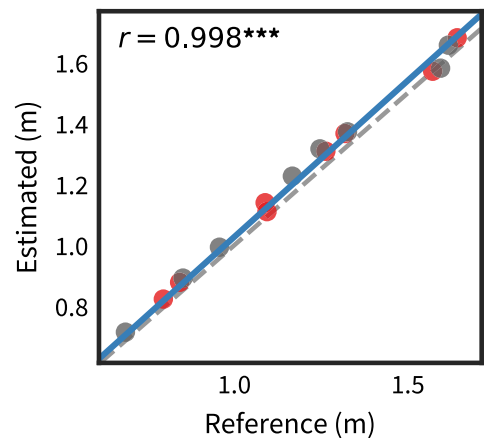

P MLD

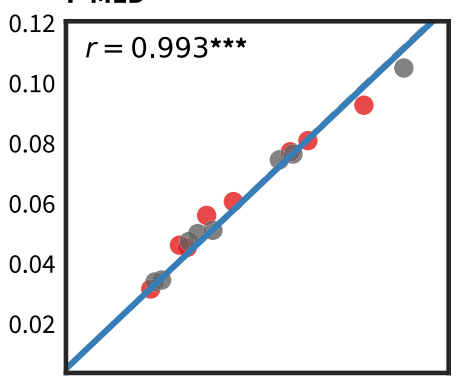

NP MLD

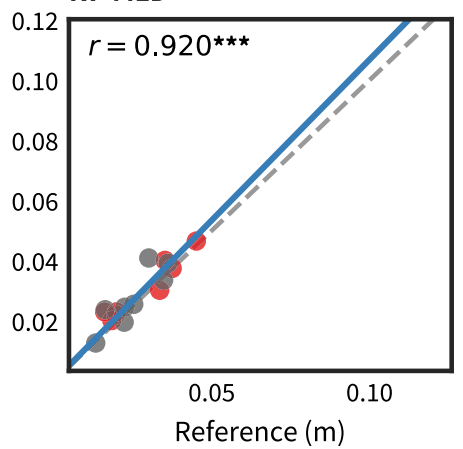

P MVD

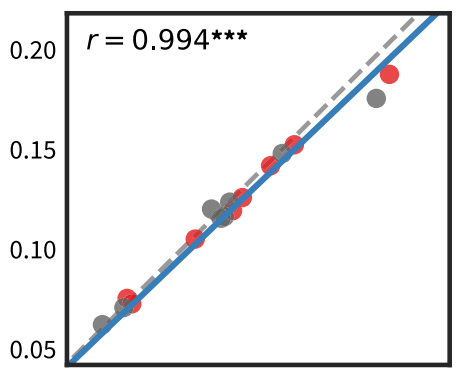

NP MVD

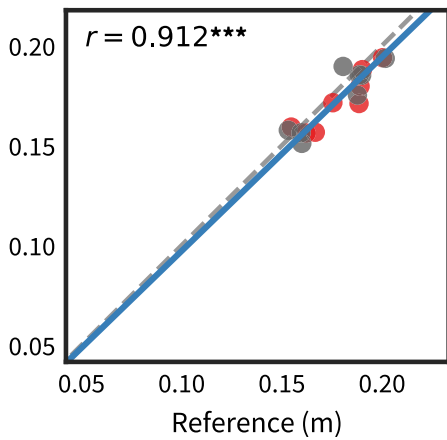

Figure 5. Regression Plots for three gait metrics, stride length (SL), maximum lateral displacement $(M L D)$, and maximum vertical displacement (MVD), shown for the paretic $(P)$ and nonparetic (NP) sides. Each point is the average data from one participant during either the Active (red) or Transparent (grey) condition. Regression plots show Pearson's correlation (r) between reference and algorithm estimates, with $* * *$ indicating $\mathrm{p}<.001$. Plots show linear fit (blue) as well as identity line (grey dashed) representing perfect estimation.

\section{Outcome Metrics}

Gait metrics were computed as defined above. Accuracy and precision were evaluated in terms of mean (absolute) errors between motion capture and estimated metrics and their respective standard deviations. We further computed inter-rater reliability based on intraclass correlation analysis $(I C C)$ according to the convention by McGraw and Wong (1996), evaluating agreement $\operatorname{ICC}(A, 1)$ and consistency $I C C(C, 1)$ between both measurement systems. We also computed Pearson's correlation coefficient between motion capture and estimated metrics, along with the $95 \%$ limits of agreement (LoA) between the two metrics. Lastly, we defined robustness in terms of detected versus actual ZUPT events. A ZUPT event was considered correctly detected if it fell within a ground truth window defined based on optical motion capture trajectories. That is, a ground truth ZUPT instance was defined as long as the direction of progression trajectory remained (quasi) stationary, which was considered to be the case if the difference between subsequent sample values remained below a threshold value of $1 \mathrm{~mm}$.

\section{Results}

Figure 5 and Table 2 illustrate the results for the three computed gait metrics, separated by paretic and nonparetic sides. Furthermore, Table 3 summarizes the statistical outcome metrics based on a total of 227 (P) and 220 (NP) usable strides for which our approach correctly detected ZUPTs in all cases for both sides. Following the interpretation guidelines from Cicchetti (1994), we observed excellent inter-rater reliability both in terms of agreement $(0.84<\operatorname{ICC}(A, 1)<1.00)$ and consistency $(0.86<\operatorname{ICC}(C, 1)<1.00)$ across all metrics (Table 3$)$. Following, we list the main outcomes for each gait metric in more detail. 


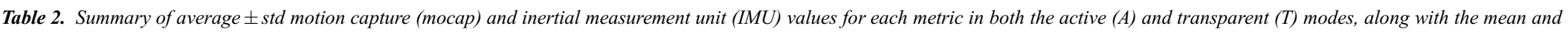
percent error for between ground truth motion capture and IMU-estimated values

\begin{tabular}{|c|c|c|c|c|c|c|c|c|c|}
\hline & & \multicolumn{2}{|c|}{ Mocap value $(\mathrm{cm})$} & \multicolumn{2}{|c|}{ IMU value $(\mathrm{cm})$} & \multicolumn{2}{|c|}{ Mean error $(\mathrm{cm})$} & \multicolumn{2}{|c|}{ Percent error $(\%)$} \\
\hline & & $\mathrm{P}$ & NP & $\mathrm{P}$ & NP & $\mathrm{P}$ & NP & $\mathrm{P}$ & NP \\
\hline \multirow[t]{2}{*}{ SL } & $\mathrm{A}$ & $120.2 \pm 29.0$ & $120.2 \pm 28.9$ & $122.6 \pm 29.8$ & $123.5 \pm 28.7$ & $2.4 \pm 1.3$ & $3.3 \pm 1.6$ & $1.9 \pm 0.7$ & $2.9 \pm 1.5$ \\
\hline & $\mathrm{T}$ & $117.7 \pm 31.5$ & $118.1 \pm 31.4$ & $120.3 \pm 32.5$ & $121.9 \pm 31.0$ & $2.6 \pm 1.1$ & $3.8 \pm 2.3$ & $2.1 \pm 0.6$ & $3.5 \pm 1.9$ \\
\hline \multirow[t]{2}{*}{ MLD } & A & $6.0 \pm 2.2$ & $2.8 \pm 1.0$ & $6.1 \pm 1.9$ & $3.0 \pm 0.9$ & $0.1 \pm 0.4$ & $0.2 \pm 0.3$ & $3.3 \pm 6.4$ & $10.9 \pm 14.4$ \\
\hline & $\mathrm{T}$ & $5.9 \pm 2.5$ & $2.5 \pm 0.8$ & $5.9 \pm 2.3$ & $2.7 \pm 0.9$ & $-0.0 \pm 0.3$ & $0.2 \pm 0.4$ & $1.1 \pm 4.4$ & $9.9 \pm 19.1$ \\
\hline \multirow[t]{2}{*}{ MVD } & A & $12.6 \pm 4.0$ & $17.8 \pm 1.5$ & $12.2 \pm 3.6$ & $17.2 \pm 1.3$ & $-0.4 \pm 0.5$ & $-0.6 \pm 0.6$ & $-2.7 \pm 3.1$ & $-3.5 \pm 3.4$ \\
\hline & $\mathrm{T}$ & $11.9 \pm 4.0$ & $17.8 \pm 1.7$ & $11.6 \pm 3.5$ & $17.4 \pm 1.6$ & $-0.3 \pm 0.8$ & $-0.3 \pm 0.7$ & $-1.6 \pm 4.5$ & $-1.8 \pm 3.7$ \\
\hline
\end{tabular}

Abbreviations: MLD, maximum lateral displacement; MVD, maximum vertical displacement; NP, nonparetic; P, paretic; SL, stride length. 
Table 3. Summary of statistical differences between motion capture ground truth and inertial measurement unit (IMU)-based estimates for the Active $(A)$ and Transparent $(T)$ conditions, including Pearson's correlation coefficient $(r ; * * \mathrm{p}<.01$,

$* * * \mathrm{p}<.001)$, limits of agreement $($ LoA;95\% LoA), and inter-class correlation coefficients for both agreement $(I C C(A, 1))$ and consistency $(\operatorname{ICC}(A, 1))$

\begin{tabular}{|c|c|c|c|c|c|c|c|c|c|}
\hline & & \multicolumn{2}{|c|}{$r$} & \multicolumn{2}{|c|}{ LoA (cm) } & \multicolumn{2}{|c|}{$\operatorname{ICC}(\mathrm{A}, 1)$} & \multicolumn{2}{|c|}{$\mathrm{ICC}(\mathrm{C}, 1)$} \\
\hline & & $\mathrm{P}$ & NP & $\mathrm{P}$ & $\mathrm{NP}$ & $\mathrm{P}$ & $\mathrm{NP}$ & $\mathrm{P}$ & $\mathrm{NP}$ \\
\hline \multirow[t]{2}{*}{ SL } & $\mathrm{A}$ & $0.999 * * *$ & $0.998 * * *$ & $(-0.12,4.92)$ & $(0.06,6.47)$ & 0.996 & 0.993 & 0.999 & 0.998 \\
\hline & $\mathrm{T}$ & $1.000 * * *$ & $0.997 * * *$ & $(0.37,4.77)$ & $(-0.67,8.34)$ & 0.997 & 0.991 & 0.999 & 0.997 \\
\hline \multirow[t]{2}{*}{ MLD } & A & $0.990 * * *$ & $0.961 * * *$ & $(-0.63,0.86)$ & $(-0.35,0.78)$ & 0.984 & 0.942 & 0.983 & 0.956 \\
\hline & $\mathrm{T}$ & $0.996^{* * *}$ & $0.880 * *$ & $(-0.61,0.60)$ & $(-0.61,1.09)$ & 0.993 & 0.852 & 0.992 & 0.868 \\
\hline \multirow[t]{2}{*}{ MVD } & A & $0.997 * * *$ & $0.917 * *$ & $(-1.42,0.56)$ & $(-1.81,0.54)$ & 0.987 & 0.847 & 0.991 & 0.912 \\
\hline & $\mathrm{T}$ & $0.990 * * *$ & $0.917 * *$ & $(-1.81,1.15)$ & $(-1.64,0.96)$ & 0.979 & 0.908 & 0.980 & 0.916 \\
\hline
\end{tabular}

Abbreviations: MLD, maximum lateral displacement; MVD, maximum vertical displacement; NP, nonparetic; P, paretic; SL, stride length.

\section{Stride length}

Among the metrics reported in this study, stride length was most accurately reconstructed, with mean estimation errors less than $2.6 \pm 1.1 \mathrm{~cm}(\mathrm{P})$ and $3.8 \pm 2.3 \mathrm{~cm}(\mathrm{NP})$ corresponding to $2.1 \pm 0.6 \%(\mathrm{P})$ and $3.5 \pm 1.9 \%(\mathrm{NP})$.

\section{Maximum lateral displacement}

Mean estimation errors for MLD were smaller than $0.11 \pm 0.38 \mathrm{~cm}(\mathrm{P})$ and $0.24 \pm 0.43 \mathrm{~cm}(\mathrm{NP})$, corresponding to $3.31 \pm 6.38 \%(\mathrm{P})$ and $10.9 \pm 14.5 \%(\mathrm{NP})$.

\section{Maximum vertical displacement}

Mean estimation errors for MVD were smaller than $-0.43 \pm 0.51 \mathrm{~cm}(\mathrm{P})$ and $-0.64 \pm 0.60 \mathrm{~cm}(\mathrm{NP})$, corresponding to $-2.68 \pm 3.07 \%(\mathrm{P})$ and $-3.46 \pm 3.36 \%(\mathrm{NP})$.

\section{Real-Time Gait Metric Response to Exosuit Assistance}

While the previous validation experiment demonstrated our method's robustness and accuracy, the feasibility of monitoring gait outside the lab would enable our long-term goal of deploying community-based rehabilitation technologies. As exosuits can instantaneously switch between providing assistance and being transparent to the wearer, monitoring gait reliably and continuously could make exosuits more versatile. Allowing them to provide assistance only when needed by the wearer could enable training with higher intensity while maintaining gait of a certain quality. To demonstrate how monitoring the efficacy of exosuit assistance might shape such training scenarios in the future, we conducted a case study $(N=1)$ mimicking more real-world conditions. To this end, we asked participant $\mathrm{S} 3$ to return for an additional visit during which we monitored gait metric response to exosuit assistance in a community environment.

\section{Experimental Overview}

The participant walked five laps around a predetermined $150 \mathrm{~m}$ circuit in a well-trafficked plaza near Harvard University (Figure 6). For the first two laps, the participant walked wearing an exosuit without obtaining assistance (transparent). Previous studies have shown that walking with an exosuit in the transparent condition is a close representation of a person's natural, baseline walking capability (Awad et al., 2020). At the beginning of the third lap, a researcher activated assistance. The participant then underwent one lap allowing adaptation to the assistance, and two more laps walking with the exosuit active. Performing this experiment as a loop rather than as a timed walk over straight pathways ensured that the active and inactive conditions took place over identical terrain. The participant was instructed to walk at a comfortable speed she could maintain over the entire trial. Medical clearance and a signed 


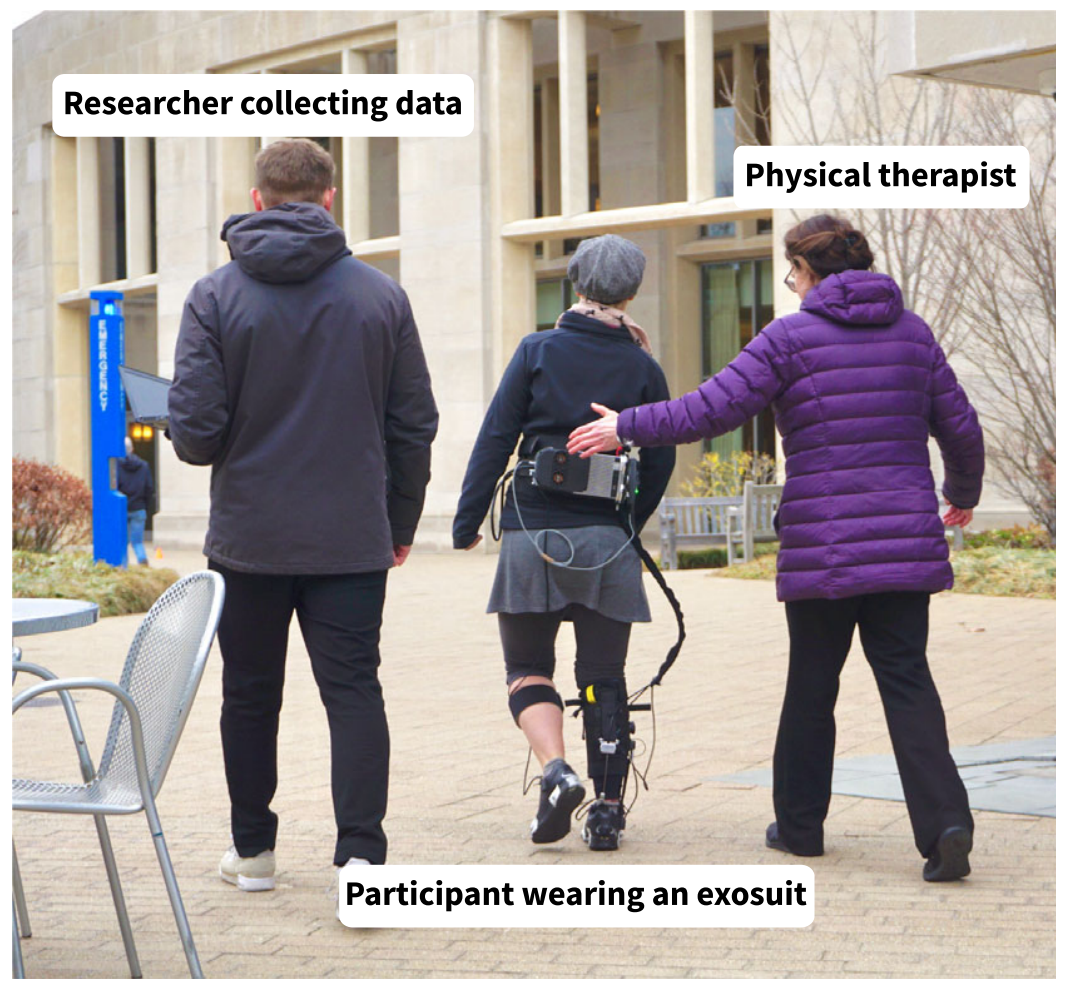

Figure 6. Experimental setup for collecting gait metric data during community-based walking. The participant was guided by a physical therapist for a total of five laps around a well-trafficked plaza at Harvard University. Sensor data were streamed via Bluetooth to a nearby computer, carried by a researcher following the participant.

informed consent form approved by the Harvard University Human Subjects Review Board were obtained for this participant.

\section{Outcome Metrics}

An indicator of walking impairment poststroke (Von Schroeder et al., 1995), we chose stride length as an outcome metric to compare the effect of walking with exosuit assistance (active) to no exosuit assistance (transparent). To test for statistical significance in stride length between the two suit conditions, we performed nonparametric analysis of variance (Wilcoxon Rank Sum test) as the obtained results were partially non-normally distributed.

\section{Results}

We collected a total of 498 strides. Significant differences comparing exosuit assistance (active) to no assistance (transparent) were captured by the algorithm during our in-lab validation experiment, showing an increase of $13.9 \mathrm{~cm}(\mathrm{P})$ and $13.7 \mathrm{~cm}(\mathrm{NP})$ for stride length (both $p<.001)$. Similarly, during outdoor testing, after adaptation to exosuit assistance, we saw a significant increase of $7.9 \mathrm{~cm}(\mathrm{P})$ and $7.3 \mathrm{~cm}$ (NP) for stride length compared to no suit assistance (both $p<.001$ ). Figure 7 shows the effect of exosuit assistance on poststroke gait, illustrated for stride length. 

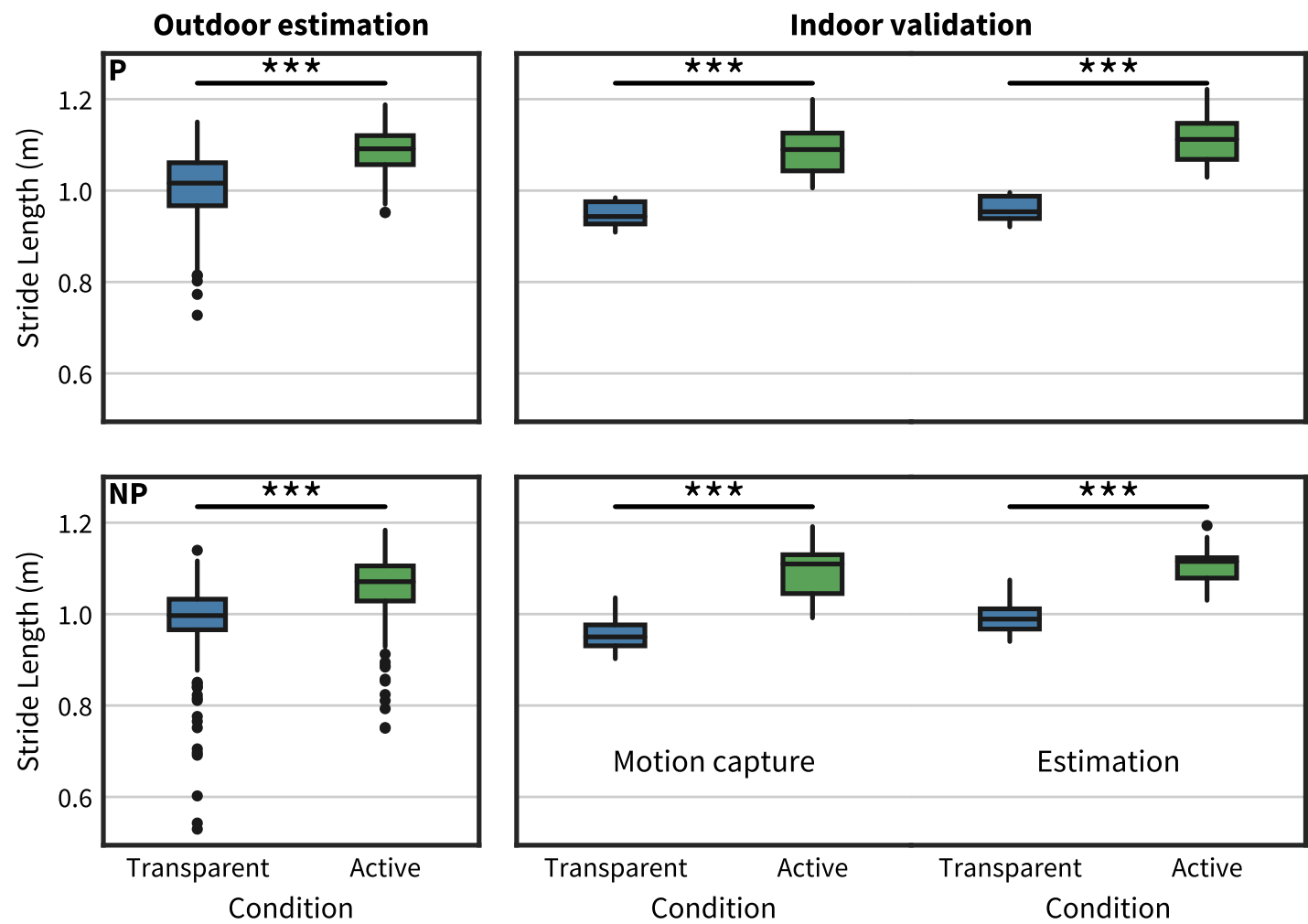

Figure 7. Gait metric response in poststroke overground walking with and without exosuit assistance shown for $P$ and NP stride lengths. The inertial measurement unit-based monitoring algorithm presented here detected significant differences between active and transparent modes $(* * * \mathrm{p}<.001)$.

\section{Discussion}

In this work, we used exosuit-integrated inertial sensors for gait metric estimation in chronic poststroke overground walking. To this end, we implemented a double integration algorithm with linear drift compensation. The main contribution of this work lies within a new form of ZUPT detection approach that informs the algorithm when to perform integration resets and correct sensor drift. That is, instead of relying on ipsilateral detection thresholds, our method defines ZUPT instances contralaterally, utilizing information from both feet. Confirming our hypothesis, we demonstrated this approach to be highly robust to irregular walking conditions poststroke.

Our in-lab validation showed excellent agreement between estimation and ground truth optical motion capture. In comparison, being among the most accurate methods found in the literature, Trojaniello et al. (2014) reported stride length in hemiparetic walking with comparable mean absolute errors of 3\%. Visi et al. (2017) showed similar outcomes for stride length in hemiparetic gait, reporting comparable to higher mean errors of $2.3 \pm 1.7 \%(\mathrm{NP})$ and $5.7 \pm 2.0 \%(\mathrm{P})$, respectively, however considering only a small sample size of $N=1$. Though data on stroke populations are limited, outcomes reported for other impaired populations may allow more extended comparisons. Hannink et al. (2016) reviewed multiple studies estimating stride length in elderly and impaired populations, reporting (relative) precisions between $6.1 \mathrm{~cm}$ and $8.4 \mathrm{~cm}(5-11 \%)$, with mean errors close to zero.

Regarding MLD, the relatively high percentage errors we observed - particularly on the NP side — can be explained by the very small absolute lateral displacements of $5 \mathrm{~cm}(\mathrm{P})$ and $2 \mathrm{~cm}(\mathrm{NP})$, which mean even small errors appear quite substantial. Moreover, errors of a few $\mathrm{mm}$ are approaching the smallest distance resolvable with the $9 \mathrm{~mm}$ reflective markers used for this study. Still, comparing these outcomes to swing 
width errors in Parkinsonian gait $(0.15 \pm 2.13 \mathrm{~cm})$ (Mariani et al., 2013), we observe comparable mean estimation errors with noticeably improved precision for both sides.

Lastly, estimation errors for MVD are lower than Hannink et al. (2017), who tested various reconstruction methods on a clinical benchmark dataset with accuracies of $-0.84 \pm 3.98 \mathrm{~cm}$. Moreover, our errors are comparable to Kitagawa and Ogihara (2016) $(0.2 \pm 0.7 \mathrm{~cm})$ and substantially lower than Mariani et al. (2012) $(4.2 \pm 2.2 \mathrm{~cm})$, both reported for healthy populations.

In summary, we found improvements on previously reported precisions, suggested by smaller error variance across metrics. Mean estimation errors were comparable or lower than reported for MLD and MVD, while showing slightly biased error distributions for stride length. A comparison to the method published in Skog et al. (2010) on the data set presented here (Supplementary Table S1) indicates the same. While difficult to confirm without an extended analysis, the small error variance may potentially come from a difference in reliability in detecting ZUPTs between our method and others. False positives (i.e., ZUPTs during swing) would likely result in underestimated positions by cutting the integration period artificially short. Conversely, false negatives (i.e., missed ZUPTs) could lead to overestimated positions by letting the integration accumulate for too long. Combined, these inaccuracies may result in mean error distributions centered around zero, but with increased variability compared to a method that misses no ZUPTs. A future analysis retuning both the window size and detection threshold from Skog et al. (2010) to poststroke walking may provide further insight regarding this hypothesized relationship between missed or false ZUPTs and estimation errors. Considering that we enforce ZUPTs at single instances as opposed to over periods of time could explain the slightly biased errors distributions observed in some cases, as this allows drift to accumulate for longer periods of time. While in theory accounted for by the drift compensation, this can become more problematic if bias instabilities - potentially caused by inaccurate orientation estimates or genuine IMU bias fluctuations - invalidate the assumption of linear velocity drift (Woodman, 2007). Incorporating higher-order drift functions that potentially better capture these effects may increase accuracy in future work. In addition, we expect a certain degree of residual error due to factors such as misalignment between the IMU coordinate frame origin and the coordinate frame origin approximated by the optical markers (see Figure 4), soft tissue artifacts or maximum achievable resolutions given optical marker sizes of up to $9 \mathrm{~mm}$.

Gait training is an integral component of recovery after a stroke. While effective, current clinical standards may lack the precision and frequency to monitor small improvements in walking during and after therapy. As wearable devices such as soft exosuits encourage community or at-home rehabilitation, and as the field of gait therapy continues to encourage telemedicine, monitoring gait quality becomes even more important. Devices capable of measuring both short- and long-term changes in walking will inform not only the development and application of exoskeletons, but also help expand current therapeutic practices out of the clinic.

With respect to clinical applicability, an important assessment criterion is a method's sensitivity to changes in gait characteristics over time. Assessing long-term gait changes may provide insights to rehabilitation progression or general mobility declines, while monitoring the immediate response to intervention approaches such as exosuit assistance may help inform treatment implementations. As particularly within a session such differences can be small, gait monitoring systems require measurement precision high enough to resolve subtle but clinically meaningful outcomes reliably. In this context, Hannink et al. (2016) argued that for sufficient resolution, error standard deviations need to be less than half the true effect magnitude. Moreover, a reasonable evaluation benchmark could be an algorithm's capability of resolving minimally detectable changes (MDCs), representing important reference values in everyday clinical assessment. Kesar et al. (2011) for example, reported paretic within-session step length MDCs of $2.62 \mathrm{~cm}$ in poststroke (treadmill) walking. For stride length, though not explicitly reported, this could suggest standard deviation requirements in ranges lower than $\pm 2.6 \mathrm{~cm}$, if we assume stride length to be about twice step length. Notably, people poststroke typically exhibit less variability on a treadmill than overground, shrinking treadmill-derived MDCs and making them more difficult to resolve. For betweensession assessment, recent work by Geiger et al. (2019) found stride length MDCs of around $11.96 \mathrm{~cm}$ in poststroke gait which — by the same reasoning — would require standard deviations in ranges lower than 
$6 \mathrm{~cm}$. Standard deviations of less than $1.3 \mathrm{~cm}(\mathrm{P})$ and $2.3 \mathrm{~cm}(\mathrm{NP})$ achieved in this work would be sufficiently small to meet these requirements in both cases.

As a complement to our in-lab validation, our use-case experiment further demonstrated our algorithm's robustness in conditions typical to more pedestrian situations, such as negotiating small obstacles in the walkway and navigating around passersby. The ability to reliably monitor wearer response to exosuit assistance in such community-like settings could help establish new exosuit-assisted gait training paradigms. Indeed, recent work on control individualization (Siviy et al., 2020) underscores how people poststroke can be sensitive to changes in exosuit assistance. Future work in this domain could explore gait metrics as objective functions to automatically tune assistance parameters based on individual wearer response. By closing the loop between assessment of gait and intervention, exosuits may thereby assist throughout the whole spectrum of poststroke rehabilitation. Still, the feasibility study presented here contains data from only a single participant. Analyses of how well the exosuit improves gait in people poststroke with more statistical power have been conducted in the lab (Awad et al., 2017; 2020), but future work may wish to repeat those analyses in the community.

Limitations of this study may guide further directions for future work. To that end, incorporating a simple model of the foot in swing could provide more granular information about foot clearance, instead of ankle clearance only. Minimal toe clearance is particularly interesting to clinicians, as it is an indicator of tripping risk in stroke survivors (Begg et al., 2014). In addition, measures such as step length asymmetry are clinically relevant, but require more information about the relative position of either foot than is available from the present pair of foot-mounted IMUs. In prior work, this has been addressed by using different sets of IMUs (Moore et al., 2017; Wang et al., 2018), mounting an IMU to the pelvis (Köse et al., 2012), or by using additional sensors (Meulen et al., 2016). Moreover, while estimation error was small on both sides, accuracy differences between the paretic and nonparetic side could be subject to further investigation in the future. That we saw higher estimation errors on the NP side may be the result of higher variation in $\mathrm{P}$ side toe-off detection due to $\mathrm{P}$ side walking instability. More specifically, as nonparetic ZUPTs require information from the contralateral (paretic) side, paretic side instability may manifest as slightly poorer ZUPT estimates on the nonparetic side. This is a primary distinction from methods such as Visi et al. (2017), which rely on purely ipsilateral information for ZUPT detection. Exploring error sensitivity to nonlinear drift functions, as indicated previously, may provide more conclusive information in this regard. Lastly, the method presented here requires IMUs that can provide orientation estimates in order to calculate accelerations in the global frame. Future work exploring how orientation estimates from lower-cost IMUs influence performance would be valuable in assessing our method's applicability across different hardware setups.

Acknowledgments. We would like to thank Mr Jonathan Foster, Mr Jack Eiel, and Ms Lauren Bizarro for their contributions to data collection. We would also like to thank the participants who donated their time to this study.

Funding Statement. Research reported in this publication was supported by the National Institutes of Health under award number BRG R01HD088619. This material is based upon work supported by the National Science Foundation Graduate Research Fellowship under Grant No. DGE1144152. This material is based upon work supported by the National Science Foundation under Grant No. CMMI-1925085. This work was supported by American Heart Association grant AHA 18TPA34170171.

Competing Interests. Patents describing the exosuit components documented in this article have been filed with the U.S. Patent Office. C.S., J.B, N.K., and C.J.W. are authors on one or more of those patents or patent applications. Harvard University has entered into a licensing and collaboration agreement with ReWalk Robotics. C.J.W. is a paid consultant for ReWalk Robotics. The other authors declare that they have no competing interests.

Authorship Contributions. P.A., C.S., and C.J.W. designed the study. P.A. and J.B. designed the double integration framework. J.B., N.K., and C.S. provided feedback on the double integration framework. P.A, C.S., and D.K.C. collected and processed data. P.A., C.S. wrote the first draft, and approved the final version of the manuscript. T.D.E., L.N.A., and C.J.W. revised the manuscript and approved the final version.

Data Availability Statement. The data supporting the main conclusions of this manuscript are located within the manuscript. Additional data are available upon request (C.J.W., walsh@seas.harvard.edu). 
Ethical Standards. The authors assert that all procedures contributing to this work comply with the ethical standards of the relevant national and institutional committees on human experimentation and with the Helsinki Declaration of 1975, as revised in 2008.

Supplementary Materials. To view supplementary material for this article, please visit http://doi.org/10.1017/wtc.2020.11.

\section{References}

Awad LN, Bae J, O'Donnell K, De Rossi SMM, Hendron K, Sloot LH, Kudzia P, Allen S, Holt KG, Ellis TD and Walsh CJ (2017) A soft robotic exosuit improves walking in patients after stroke. Science Translational Medicine 9(400), eaai9084. https:// doi.org/10.1126/scitranslmed.aai9084.

Awad LN, Kudzia P, Revi DA, Ellis TD and Walsh CJ (2020) Walking faster and farther with a soft robotic exosuit: Implications for poststroke gait assistance and rehabilitation. IEEE Open Journal of Engineering in Medicine and Biology 1, 108-115. https:// doi.org/10.1109/OJEMB.2020.2984429.

Bae J, Siviy C, Rouleau M, Menard N, Odonnell K, Geliana I, Athanassiu M, Ryan D, Bibeau C, Sloot L, Kudzia P, Ellis T, Awad L and Walsh CJ (2018) A lightweight and efficient portable soft exosuit for paretic ankle assistance in walking after stroke. In 2018 IEEE International Conference on Robotics and Automation (ICRA). Brisbane, AU: IEEE, pp. 2820-2827. Available at https://doi.org/10.1109/ICRA.2018.8461046 (accessed Feb 20, 2020).

Begg RK, Tirosh O, Said CM, Sparrow WA, Steinberg N, Levinger P and Galea MP (2014) Gait training with real-time augmented toe-ground clearance information decreases tripping risk in older adults and a person with chronic stroke. Frontiers in Human Neuroscience 8, 243.

Benjamin EJ, Muntner P, Alonso A, Bittencourt MS, Callaway CW, Carson AP, Chamberlain AM, Chang AR, Cheng S, Das SR, Delling FN, Djousse L, Elkind MSV, Ferguson JF, Fornage M, Jordan LC, Khan SS, Kissela BM, Knutson KL, Kwan TW, Lackland DT, Lewis TT, Lichtman JH, Longenecker CT, Loop MS, Lutsey PL, Martin SS, Matsushita K, Moran AE, Mussolino ME, O'Flaherty M, Pandey A, Perak AM, Rosamond WD, Roth GA, Sampson UKA, Satou GM, Schroeder EB, Shah SH, Spartano NL, Stokes A, Tirschwell DL, Tsao CW, Turakhia MP, VanWagner LB, Wilkins JT, Wong SS, Virani SS and On behalf of the American Heart Association Council on Epidemiology and Prevention Statistics Committee and Stroke Statistics Subcommittee (2019) Heart disease and stroke statistics - 2019 update: A report from the American Heart Association. Circulation 139(10), e56-e528. https://doi.org/10.1161/CIR.0000000000000659.

Benoussaad M, Sijobert B, Mombaur K and Coste CA (2016) Robust foot clearance estimation based on the integration of footmounted IMU acceleration data. Sensors 16(1), 12.

Cicchetti DV (1994) Guidelines, criteria, and rules of thumb for evaluating normed and standardized assessment instruments in psychology. Psychological Assessment 6(4), 284.

Eng JJ and Tang PF (2007) Gait training strategies to optimize walking ability in people with stroke: A synthesis of the evidence. Expert Review of Neurotherapeutics 7(10), 1417-1436. https://doi.org/10.1586/14737175.7.10.1417.

Ferrari A, Ginis P, Hardegger M, Casamassima F, Rocchi L and Chiari L (2016) A mobile Kalman-filter based solution for the real-time estimation of spatio-temporal gait parameters. IEEE Transactions on Neural Systems and Rehabilitation Engineering 24(7), 764-773. https://doi.org/10.1109/TNSRE.2015.2457511.

Foxlin E (2005) Pedestrian tracking with shoe-mounted inertial sensors. IEEE Computer Graphics and Applications 25(6), 38-46.

Geiger M, Supiot A, Pradon D, Do M-C, Zory R and Roche N (2019) Minimal detectable change of kinematic and spatiotemporal parameters in patients with chronic stroke across three sessions of gait analysis. Human Movement Science 64, 101-107. https:// doi.org/10.1016/j.humov.2019.01.011.

Hannink J, Kautz T, Pasluosta CF, Barth J, Schülein S, Gaßmann K-G, Klucken J and Eskofier BM (2016) Stride length estimation with deep learning. IEEE Journal of Biomedical and Health Informatics 22(2), 354-362. https://doi.org/10.1109/ JBHI.2017.2679486.

Hannink J, Ollenschläger M, Kluge F and Roth N (2017) Benchmarking foot trajectory estimation methods for mobile gait analysis. Sensors 17(9), 1940. https://doi.org/doi:10.3390/s17091940.

Hickey A., Horgan F, O’Neill D and McGee H (2012) Community-based poststroke service provision and challenges: A national survey of managers and inter-disciplinary healthcare staff in Ireland. BMC Health Services Research 12(1), 111. https://doi. org/10.1186/1472-6963-12-111.

Jayaraman A, O'Brien MK, Madhavan S, Mummidisetty CK, Roth HR, Hohl K, Tapp A, Brennan K, Kocherginsky M, Williams KJ, Takahashi H and Rymer WZ (2019) Stride management assist exoskeleton versus functional gait training in stroke. Neurology 92(3), e263-e273. https://doi.org/10.1212/WNL.0000000000006782.

Jim AR (2009) A comparison of pedestrian dead-reckoning algorithms using a low-cost MEMS IMU. In 2009 IEEE International Symposium on Intelligent Signal Processing. Budapest: IEEE, pp. 37-42. Available at https://doi.org/10.1109/ WISP.2009.5286542 (accessed Jan 6, 2020).

Kempski K, Awad LN, Buchanan TS, Higginson JS and Knarr BA (2018) Dynamic structure of lower limb joint angles during walking poststroke. Journal of Biomechanics 68, 1-5. https://doi.org/10.1016/j.jbiomech.2017.12.019.

Kesar TM, Binder-Macleod SA, Hicks GE and Reisman DS (2011) Minimal detectable change for gait variables collected during treadmill walking in individuals poststroke. Gait \& Posture 33(2), 314-317.

Kitagawa $\mathbf{N}$ and Ogihara N (2016) Estimation of foot trajectory during human walking by a wearable inertial measurement unit mounted to the foot. Gait \& Posture 45, 110-114. https://doi.org/10.1016/j.gaitpost.2016.01.014. 
Köse A, Cereatti A and Della Croce U (2012) Bilateral step length estimation using a single inertial measurement unit attached to the pelvis. Journal of NeuroEngineering and Rehabilitation 9(1), 9. https://doi.org/10.1186/1743-0003-9-9.

Langhorne P, Coupar F and Pollock A (2009) Motor recovery after stroke: A systematic review. The Lancet Neurology 8(8), 741-754. https://doi.org/10.1016/S1474-4422(09)70150-4.

Do Lee C, Folsom AR and Blair SN (2003) Physical activity and stroke risk. Stroke 34(10), 2475-2481. https://doi. org/10.1161/01.STR.0000091843.02517.9D.

Linden T., Blomstrand C and Skoog I (2007) Depressive disorders after 20 months in elderly stroke patients. Stroke 38(6), 1860-1863. https://doi.org/10.1161/STROKEAHA.106.471805.

Mariani B, Hoskovec C, Rochat S, Büla C, Penders J and Aminian K (2010) 3D gait assessment in young and elderly subjects using foot-worn inertial sensors. Journal of Biomechanics 43(15), 2999-3006. https://doi.org/10.1016/j.jbiomech.2010.07.003.

Mariani B, Jiménez MC, Vingerhoets FJG and Aminian K (2013) On-shoe wearable sensors for gait and turning assessment of patients with Parkinson's disease. IEEE Transactions on Biomedical Engineering 60(1), 155-158. https://doi.org/10.1109/ TBME.2012.2227317.

Mariani B, Rochat S, Büla CJ and Aminian K (2012) Heel and toe clearance estimation for gait analysis using wireless inertial sensors. IEEE Transactions on Biomedical Engineering 59(12 PART2), 3162-3168. https://doi.org/10.1109/TBME.2012.2216263.

McCain EM, Dick TJM, Giest TN, Nuckols RW, Lewek MD, Saul KR and Sawicki GS (2019) Mechanics and energetics of poststroke walking aided by a powered ankle exoskeleton with speed-adaptive myoelectric control. Journal of NeuroEngineering and Rehabilitation 16(1), 57. https://doi.org/10.1186/s12984-019-0523-y.

McGraw KO and Wong SP (1996) Forming inferences about some intraclass correlation coefficients. Psychological Methods 1(1), 30.

van Meulen FB, Weenk D, Buurke JH, van Beijnum B-JF and Veltink PH (2016) Ambulatory assessment of walking balance after stroke using instrumented shoes. Journal of NeuroEngineering and Rehabilitation 13(1), 48. https://doi.org/10.1186/ s12984-016-0146-5.

Moore SA, Hickey A, Lord S, Del Din S, Godfrey A and Rochester L (2017) Comprehensive measurement of stroke gait characteristics with a single accelerometer in the laboratory and community: a feasibility, validity and reliability study. Journal of NeuroEngineering and Rehabilitation 14(1), 130. https://doi.org/10.1186/s12984-017-0341-z.

Olney SJ and Richards C (1996) Hemiparetic gait following stroke. Part I: Characteristics. Gait \& Posture 4(2), 136-148. https:// doi.org/10.1016/0966-6362(96)01063-6.

Paolucci S (2008) Epidemiology and treatment of poststroke depression. Neuropsychiatric Disease and Treatment 4(1), $145-154$. Available at http://www.ncbi.nlm.nih.gov/pubmed/18728805 and http://www.pubmedcentral.nih.gov/articlerender.fcgi?artid= PMC2515899.

Paolucci S, Grasso MG, Antonucci G, Bragoni M, Troisi E, Morelli D, Coiro P, De Angelis D and Rizzi F (2001) Mobility status after inpatient stroke rehabilitation: 1-Year follow-up and prognostic factors. Archives of Physical Medicine and Rehabilitation 82(1), 2-8. https://doi.org/10.1053/apmr.2001.18585.

Porciuncula F, Roto AV, Kumar D, Davis I, Roy S, Walsh CJ and Awad LN (2018) Wearable movement sensors for rehabilitation: A focused review of technological and clinical advances. $P M \& R$ 10(9), S220-S232. https://oi.org/10.1016/j. pmrj.2018.06.013.

Rampp A, Barth J, Schülein S, Gaßmann KG, Klucken J and Eskofier BM (2015) Inertial sensor-based stride parameter calculation from gait sequences in geriatric patients. IEEE Transactions on Biomedical Engineering 62(4), 1089-1097. https:// doi.org/10.1109/TBME.2014.2368211.

Rebula JR, Ojeda LV, Adamczyk PG and Kuo AD (2013) Measurement of foot placement and its variability with inertial sensors. Gait \& Posture 38(4), 974-980. https://doi.org/10.1016/j.gaitpost.2013.05.012.

Sabatini AM, Martelloni C, Scapellato S and Cavallo F (2005) Assessment of walking features from foot inertial sensing. IEEE Transactions on Biomedical Engineering 52(3), 486-494.

Schepers MH, van Asseldonk EHF, Baten CTM and Veltink PH (2010) Ambulatory estimation of foot placement during walking using inertial sensors. Journal of Biomechanics 43(16), 3138-3143. https://doi.org/10.1016/j.jbiomech.2010.07.039.

Schepers MH, Koopman HFJM and Veltink PH (2007) Ambulatory assessment of ankle and foot dynamics. IEEE Transactions on Biomedical Engineering 54(5), 895-902. https://doi.org/10.1109/TBME.2006.889769.

Siviy C, Bae J, Baker L, Porciuncula F, Baker T, Ellis TD, Awad LN and Walsh CJ (2020) Offline assistance optimization of a soft exosuit for augmenting ankle power of stroke survivors during walking. IEEE Robotics and Automation Letters 5(2), 828-835.

Skog I, Händel P, Nilsson JO and Rantakokko J (2010) Zero-velocity detection-An algorithm evaluation. IEEE Transactions on Biomedical Engineering 57(11), 2657-2666. https://doi.org/10.1109/TBME.2010.2060723.

Toro B, Nester CJ and Farren PC (2007) Inter- and intraobserver repeatability of the Salford gait tool: An observation-based clinical gait assessment tool. Archives of Physical Medicine and Rehabilitation 88(3), 328-332. https://doi.org/10.1016/j apmr.2006.12.030.

Trojaniello D, Cereatti A, Pelosin E, Avanzino L, Mirelman A, Hausdorff JM and Della Croce U (2014) Estimation of step-bystep spatio-temporal parameters of normal and impaired gait using shank-mounted magneto-inertial sensors: Application to elderly, hemiparetic, parkinsonian and choreic gait. Journal of NeuroEngineering and Rehabilitation 11(1), 1-12. https://doi. org/10.1186/1743-0003-11-152.

Visi F, Georgiou T, Holland S, Pinzone O, Donaldson G and Tetley J (2017) Assessing the accuracy of an algorithm for the estimation of spatial gait parameters using inertial measurement units: Application to healthy subject and hemiparetic stroke 
survivor. In Proceedings of the 4th International Conference on Movement Computing. New York, NY: ACM, pp. 1-7. https:// dl.acm.org/doi/abs/10.1145/3077981.3078034 (accessed Jan 6, 2020).

Von Schroeder HP, Coutts RD, Lyden PD, Billings E and Nickel VL (1995) Gait parameters following stroke: A practical assessment. Journal of Rehabilitation Research and Development 32, 25-25.

Walker MF, Sunnerhagen KS and Fisher RJ (2013) Evidence-based community stroke rehabilitation. Stroke 44(1), $293-297$. https://doi.org/10.1161/STROKEAHA.111.639914.

Wang L, Sun Y, Li Q and Liu T (2018) Estimation of step length and gait asymmetry using wearable inertial sensors. IEEE Sensors Journal 18(9), 3844-3851. https://doi.org/10.1109/JSEN.2018.2815700.

Winstein CJ, Stein J, Arena R, Bates B, Cherney LR, Cramer SC, Deruyter F, Eng JJ, Fisher B, Harvey RL and Lang CE (2016) Guidelines for adult stroke rehabilitation and recovery: a guideline for healthcare professionals from the American Heart Association/American Stroke Association. Stroke 476, e98-e169. https://doi.org/10.1161/STR.0000000000000098.

Woodman OJ (2007) An Introduction to Inertial Navigation. Number 696. Cambridge, UK: University of Cambridge. Available at http:/www.cl.cam.ac.uk/techreports/ (accessed Jan 6, 2020).

Zhang M, Hol JD, Slot L, Luinge H and Xsens Technologies BV (2011) Second order nonlinear uncertainty modeling in strapdown integration using MEMS IMUs. In 2011 Proceedings of the 14th International Conference on Information Fusion (FUSION). Chicago, IL: IEEE, pp. 1-7. https://ieeexplore.ieee.org/document/5977574 (accessed Aug 3, 2020).

Cite this article: Arens P, Siviy C, Bae J, Choe D. K, Karavas N, Baker T, Ellis T. D, Awad L. N and Walsh C. J (2021). Real-time gait metric estimation for everyday gait training with wearable devices in people poststroke. Wearable Technologies, 2, e2, doi:https://doi.org/10.1017/wtc.2020.11 